\section{The Next Frontier: Stem Cells and the Center for the Advancement of Science in Space}

Duane Ratliff*

\section{ABSTRACT}

The Center for the Advancement of Science in Space (CASIS) manages the International Space Station U.S. National Laboratory, supporting spacebased research that seeks to improve life on Earth. The National Laboratory is now open for use by the broad scientific community-and CASIS is the gateway to this powerful in-orbit research platform.
The Center for the Advancement of Science in Space (CASIS) is a nonprofit organization tasked by the U.S. Congress to manage, promote, and broker research on the International Space Station U.S. National Laboratory. The CASIS mission is to make this laboratory accessible in that users may discover and generate intellectual property, newfound knowledge, and new product applications that can provide value for U.S. citizens.

CASIS is responsible for stimulating use of the International Space Station to government agencies, academic institutions, and commercial entities from basic to applied research. To achieve this goal, CASIS provides flight opportuni-

Chief Operating Officer, Center for the Advancement of Science in Space Communications, Melbourne, Florida.

*(Correspondence: dratliff@iss-casis.org) ties in two manners: solicited and unsolicited proposals. At any time, a U.S. company, institution, organization, or citizen can submit a proposal through the unsolicited proposal process that will be vetted by CASIS for operational feasibility, scientific merit, and potential economic benefit to the U.S. taxpayer. As of September 2013, CASIS has received more than 40 qualified unsolicited proposals.

CASIS has recruited a Science Advisory Board that is responsible for identifying promising areas of research in life, materials, and physical sciences along with Earth observation and remote sensing. On the basis of the recommendations of the CASIS Science Advisory Board, funded solicitations are created. As of September 2013, CASIS has funded two requests for proposals in protein crystal growth and materials science while issuing four

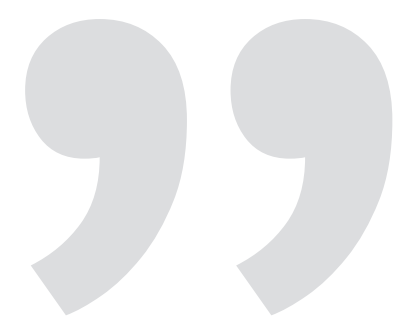

CASIS recently issued funding to seven investigators for stem cell research on the International Space Station. total proposals and two requests for information.

\section{Stem cell research on the International Space Station}

On July 25, 2013, CASIS closed a request for proposals (RFP) for stem cell research projects on the International Space Station. CASIS, overwhelmingly pleased with the amount of qualified proposals it received, confirmed the organization's confidence that both experienced and new-to-space investigators would be interested in exploiting the effects of microgravity and the space environment's unique conditions to investigate the fundamental properties of mammalian stem cells, including their ability to self-renew, proliferate, and differentiate. 


\section{CASIS offers researchers the following benefits:}

- Admission to the world's most specialized research platform-the International Space Station-for unparalleled innovation and discovery.

- Access to the experimental variables of microgravity and the extreme space environment, as well as a powerful vantage point for Earth observation.

- Support from experienced service providers who can help scientists take full advantage of the resources and facilities onboard the National Laboratory.

- Assistance in acquiring specialized hardware and software for successful space-based experiments.

- Opportunities for funding via CASIS solicitations and prize competitions.

For more information, visit www.iss-casis.org

In October, CASIS announced seven proposals that will receive funding through its stem cell RFP, totaling over $\$ 2$ million. Each experiment will use the unique microgravity environment aboard the space station to conduct experimentation that could produce tremendous health benefits for humankind-via advancements in drug screening, tissue engineering/regeneration, cell replacement therapy, and cell reprogramming

\section{The CASIS mission}

CASIS supports a broad range of research ideas, such as biomedical sciences, materials sciences, physical sciences, and re- mote sensing/Earth observation. Currently, CASIS has more than 40 projects scheduled for flight to the International Space Station that are intended to bring a tangible return on investment to the U.S. taxpayer. CASIS aims to drive scientific inquiry toward developing groundbreaking new technologies and products through carefully selecting research and funding projects, by connecting investors looking for opportunities to scientists with great ideas, and by making access to the station faster and easier. The first CASIS-sponsored investigations are scheduled to launch toward the International Space Station in December 2013.

\section{Author disclosure statement}

No competing financial interests exist. 